\title{
Olive oil consumption and risk of CHD and/or stroke: a meta-analysis of case-control, cohort and intervention studies
}

\author{
Miguel A. Martínez-González ${ }^{1,2}$, Ligia J. Dominguez ${ }^{3 *}$ and Miguel Delgado-Rodríguez ${ }^{4,5}$ \\ ${ }^{1}$ Department of Preventive Medicine and Public Health, University of Navarra, Pamplona, Spain \\ ${ }^{2}$ CIBER Fisiopatologia de la Obesidad y Nutricion (CIBERobn), Instituto de Salud Carlos III, Madrid, Spain \\ ${ }^{3}$ Geriatric Unit, Department of Internal Medicine and Geriatrics, University of Palermo, Via F. Scaduto 6/c, \\ 90144 Palermo, Italy \\ ${ }^{4}$ Division of Medicine and Health Sciences, University of Jaen, Jaen, Spain \\ ${ }^{5}$ CIBER Epidemiologia y Salud Pública (CIBERESP), Madrid, Spain \\ (Submitted 25 September 2013 - Final revision received 4 March 2014 - Accepted 4 March 2014 - First published online 28 April 2014)
}

\section{Abstract}

Increasing evidence suggests that the Mediterranean diet can reduce the risk of CVD. Olive oil is the hallmark of this dietary pattern. We conducted a meta-analysis of case-control, prospective cohort studies and a randomised controlled trial investigating the specific association between olive oil consumption and the risk of CHD (101460 participants) or stroke (38673 participants). The results of all observational studies were adjusted for total energy intake. The random-effects model assessing CHD as an outcome showed a relative risk (RR) of $0.73(95 \%$ CI $0.44,1.21)$ in case-control studies and $0.96(95 \%$ CI $0.78,1.18)$ in cohort studies for a $25 \mathrm{~g}$ increase in olive oil consumption. In cohort studies, the random-effects model assessing stroke showed a RR of $0 \cdot 74$ (95\% CI 0.60, 0.92). The random-effects model combining all cardiovascular events (CHD and stroke) showed a RR of 0.82 (95\% CI $0.70,0.96$ ). Evidence of heterogeneity was apparent for CHD, but not for stroke. Both the Egger test $(P=0.06)$ and the funnel plot suggested small-study effects. Available studies support an inverse association of olive oil consumption with stroke (and with stroke and CHD combined), but no significant association with CHD. This finding is in agreement with the recent successful results of the PREDIMED randomised controlled trial.

\section{Key words: Olive oil: Mediterranean diet: CVD: Myocardial infarction: Stroke}

A notable characteristic of the Mediterranean diet is the abundant consumption of olive oil, rich in the MUFA oleic acid. Olive oil is the typical culinary fat used in Mediterranean countries that can account for almost $20 \%$ of total energy intake. Early ecological studies have shown inverse associations between average country consumption of olive oil and the risk of $\mathrm{CVD}^{(1-3)}$ or total mortality ${ }^{(4,5)}$. In addition, there is also evidence to support that olive oil intake may have a beneficial influence on cardiovascular risk factors ${ }^{(6-9)}$. Of potential importance, some minor components of olive oil other than oleic acid have been suggested to contribute to anti-inflammatory, antioxidant and other bioactive properties that can benefit the endothelium, favourably modulate haemostatic factors and improve the stability of the arteriosclerotic plaque ${ }^{(7-9)}$. In contrast, the 'Pooling Project of Cohort Studies on Diet and Coronary Disease' showed potential harmful associations between MUFA intake and the risk of $\mathrm{CHD}^{(10)}$, thus challenging the paradigm that olive oil is cardio-protective. At least, these results do not support that olive oil may exert cardiovascular protection mainly because it is a good source of MUFA. A potential explanation for these divergent findings is that the source of MUFA (animal fat in the studies included in the Pooling Project ${ }^{(10)} v$. olive oil in the studies conducted in Mediterranean countries) may differentially influence the association between MUFA intake and the risk of CHD. Thus, there are several reasons to systematically assess whether olive oil consumption exerts protection against CHD and/or stroke.

Several case-control and prospective cohort studies have examined the association between consumption of olive oil and the risk of CHD and stroke. However, there are inconsistencies in outcomes between these studies ${ }^{(11-13)}$. The role of olive oil as the hallmark of the Mediterranean diet has been deemed to be exchangeable with other sources of monounsaturated fats to obtain also a high-quality, overall healthy food pattern ${ }^{(2)}$. The PREDIMED (PREvención con DIeta

Abbreviations: EPIC, European Prospective Investigation into Cancer and Nutrition; NOS, Newcastle-Ottawa Quality Assessment Scale; PREDIMED, PREvención con DIeta MEDiterránea (Prevention with Mediterranean Diet); RR, relative risk. 
MEDiterránea (Prevention with Mediterranean Diet)) randomised controlled trial has recently shown that a Mediterranean diet supplemented with either virgin olive oil or tree nuts was able to reduce the incidence of CVD in primary prevention among high-risk subjects ${ }^{(14)}$. Despite a significant protection for the primary combined end-point (myocardial infarction and stroke), the protection provided by the diet supplemented with extra-virgin olive oil as well with tree nuts in separate analyses was only significant for stroke, but not for myocardial infarction. Therefore, the association of olive oil consumption with the risk of myocardial infarction and/or stroke is not yet certain and more evidence is still needed, even after the PREDIMED randomised controlled trial. We conducted a meta-analysis of case-control and prospective cohort studies to quantify the association between olive oil consumption and the risk of CHD and stroke, and to assess the consistency of all published observational findings with those of the PREDIMED primary prevention trial

\section{Methods}

In accordance with the Preferred Reporting Items for Systematic Reviews and Meta-Analyses standard criteria ${ }^{(15)}$ (PRISMA checklist, see online supplementary material), we systematically searched published studies that investigated the possibility of an association between olive oil intake and cardiovascular events (myocardial infarction or stroke) in the following electronic databases: PubMed (from 1970), Embase (from 1947), the Cochrane Library (from 1993), Web of Science (from 1900) and Ovid (from 1946) up to December 2013. Relevant keywords relating to olive oil intake ('olive oil') were searched in combination with keywords relating to cardiovascular events ('cardiovascular disease' or 'cardiovascular event' or 'myocardial infarction' or 'coronary heart disease' or 'coronary artery disease' or 'ischemic heart disease' or 'angina' or 'stroke' or 'cerebrovascular disease'), and in combination with keywords relevant to the study methods ('incidence' or 'cohort' or 'follow-up' or 'case-control' or 'hazard ratio' or 'odds ratio' or 'relative risk' or 'rate ratio'). (An example of a search algorithm is included in the online supplementary material.) We also reviewed the bibliographies of the extracted articles and reviews to locate additional publications. The language of publication was not restricted.

Studies were included in the present meta-analysis if they satisfied the following criteria: the study design was casecontrol, or a cohort with prospective follow-up; the exposure of interest was olive oil consumption; the outcome was CHD event (both fatal and non-fatal myocardial infarction) or stroke (either ischaemic or haemorrhagic); the investigators reported relative risks (RR) with $95 \%$ CI for at least three quantitative categories of olive oil intake. Additionally, we excluded reviews, editorials, comments, letters without sufficient data, abstracts of meeting presentations, non-human studies and studies that did not specifically consider olive oil consumption on CHD events or stroke incidence, or for which estimates for olive oil associations were not available. Studies of other exposures (such as 'oil' or 'vegetable oil' without specification, or the whole Mediterranean dietary pattern without specification of olive oil consumption) and reporting outcomes of other diseases were also excluded. If results from a study population were reported more than once, we used the results with the longest follow-up time.

\section{Data extraction}

We extracted the following information from each study: authors; year of publication; study name; study location; study design; time of data acquisition; number of centres involved; dietary assessment method; number of FFQ items; olive oil consumption categories; median or mean intake of olive oil; validation of nutrients; validation of FFQ in study population; average duration of follow-up in years; sample size (cases and controls in case-control studies; number of participants and incident cases in cohort studies); participants' characteristics (age, sex, country of residence, percentage of smokers among the incident cases); end-points (CHD event, stroke or both); methods of outcome ascertainment; covariates adjusted in multivariable analysis; RR (95\% CI) for all categories of olive oil consumption. For studies that reported olive oil intake as portions per week or per d, we assumed that each portion was equivalent to $25 \mathrm{~g}$ olive oil. When studies had several adjustment models, we extracted those that reflected the maximum extent of adjustment for potentially confounding variables. For studies that lacked the unit of consumption (i.e. indicating only 'portions' consumed), the categories were estimated by multiplying the frequency of consumption with an average portion size according to the mean intake reported. We contacted the authors if the data of interest were not directly shown in the publications $^{(14,16)}$. To resolve discrepancies, we used group consensus and consulted a third reviewer. Data extraction was performed independently by two authors (L. J. D. and M. A. M.-G.) using a standard extraction form. The study protocol is available at http://www.unav.edu/departamento/ preventiva/publicaciones (accessed 1 December 2012).

\section{Study quality assessment}

The quality of all selected studies was assessed using the scores proposed by the modified Newcastle-Ottawa Quality Assessment Scale (NOS) ${ }^{(17)}$. The modified NOS grading standard, including case-control or cohort study, includes the following features: (1) selection, total score 4; (2) comparability, total score 2; (3) exposure (case-control)/outcome (cohort), total score 3. A high score $(>7)$ in a 0 - to 9-point scale indicates high methodological quality.

\section{Statistical analysis}

RR and 95\% CI were taken as the magnitude of association for all studies, and OR or hazard ratios were considered equivalent to RR. Any results stratified by sex were treated as two separate reports. Those articles reporting both CHD and stroke were also treated as two separate reports. Owing to the distinct cut-off points for categories in different articles, we computed a RR with $95 \%$ CI for an increased intake of 
$25 \mathrm{~g} / \mathrm{d}$ for each report. The method described by Greenland \& O'Rourke $^{(18)}$ was also used to estimate the pooled RR of an increment in $10 \mathrm{~g} / \mathrm{d}$ in olive oil consumption taking into account the reported RR for categories of olive oil consumption in each of the primary studies. Median or mean olive oil consumption in each category was used as the corresponding dose of consumption. We used preferentially the median; in those studies where the median was not reported, we used the mean. The midpoint of the upper and lower boundaries was considered as the dose of each category if the median or mean intake per category was not available. If the highest category was open-ended, the midpoint of the category was set at 1.5 times the lower boundary.

We assessed whether between-study heterogeneity existed using Cochran's $Q \chi^{2}$ test and quantified the proportion of heterogeneity with the $I^{2}$ statistic $^{(19-21)}$. The $I^{2}$ statistic describes the percentage of total variation in RR estimates that can be attributed to heterogeneity. However, we interpreted it with caution given the small number of studies included in the present meta-analysis ${ }^{(22)}$. Therefore, combined associations were estimated using both fixed and random association meta-analysis. Given that heterogeneity was apparent, we used a random-effects model (DerSimonian and Laird method). Forest plots were used to examine the overall effect. The funnel plot and the Egger test $(P<0 \cdot 1)$ were used to assess small-study effects such as publication bias. The trim and fill method was performed to identify and correct for funnel plot asymmetry arising from publication bias ${ }^{(23)}$.

We conducted analyses stratified by study design and outcome. We also sequentially repeated the meta-analysis after excluding one study at a time as sensitivity analysis. All statistical analyses were performed with Stata version 12 (StataCorp LP) using the metan and glst user-written commands ${ }^{(24)}$. All tests were two-sided with a significance level of 0.05.

\section{Results}

\section{Literature search}

Figure 1 shows the results of literature research and selection (see also online supplementary material). We identified 436 records in PubMed, 270 records in Embase, one record in the Cochrane Library, 262 records in the Web of Science and 156 records in Ovid before December 2013. After exclusion of duplicates, we screened 825 records. After exclusion of studies that did not fulfil the inclusion criteria, eighteen

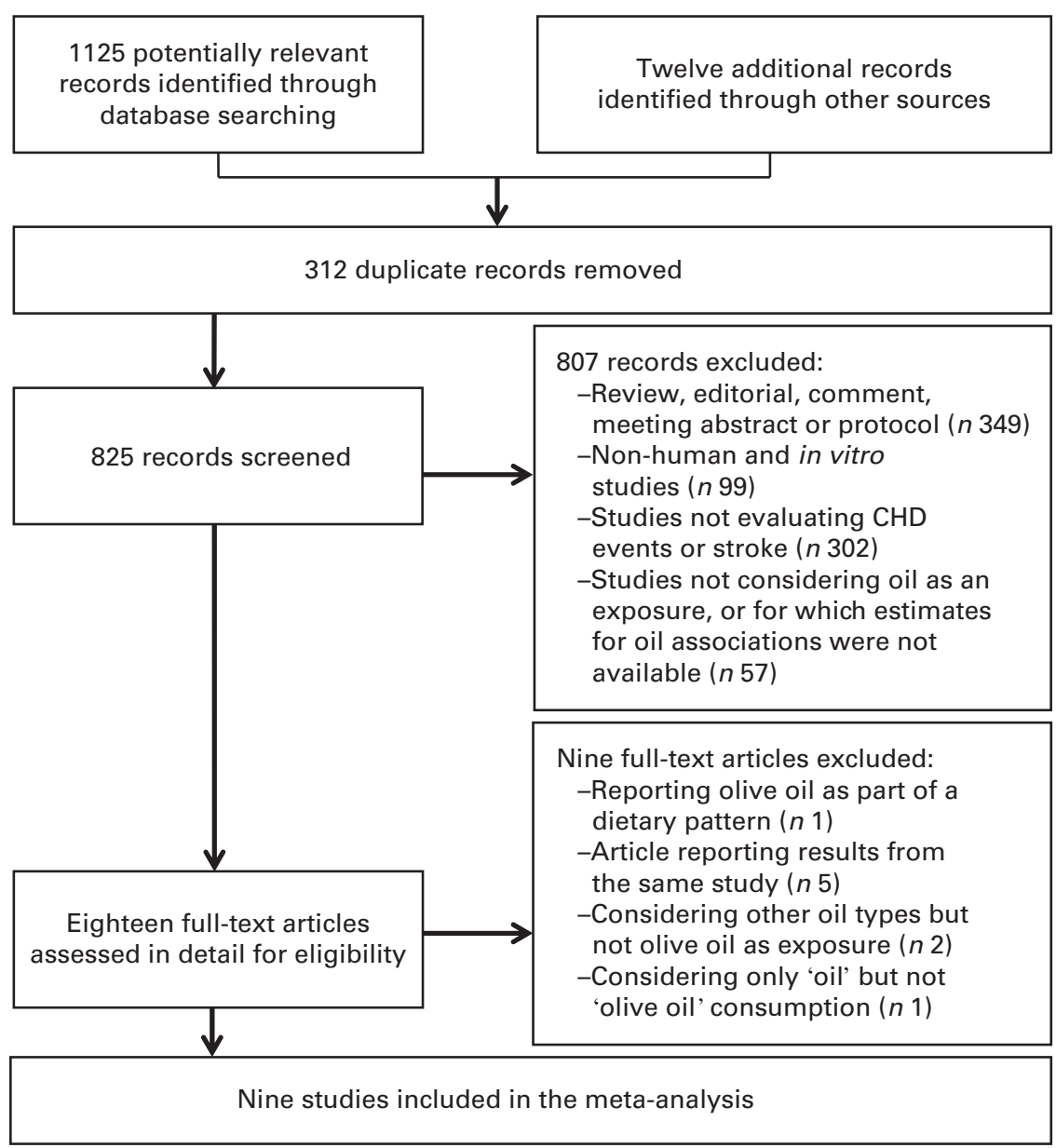

Fig. 1. Flow diagram for identification and selection process of relevant studies examining the effects of olive oil consumption and the incidence of CVD events (CHD and stroke; see online supplementary material). 
remaining articles were relevant for the present meta-analysis. The full-text review of these eighteen articles led to the exclusion of nine of them because of the reasons mentioned in Fig. 1. Finally, nine articles were available to be included in the quantitative meta-analysis (details of the included and excluded studies during the final selection process are available in the online supplementary material). Of these articles, two provided two reports; therefore, eleven estimates of effect were available to be combined. Dilis et al. ${ }^{(25)}$ included a separate report for RR for each sex, and the PREDIMED trial provided separate reports for stroke and myocardial infarction.

\section{Study characteristics}

A summary of the information extracted from the included studies is shown in Tables 1-3 (see also online supplementary material). In all studies, participants had no prior diagnoses of CVD at baseline. The present meta-analysis consisted of 101460 participants for CHD and 38673 participants for stroke. The total number of cases of CHD and controls in case-control studies were 1526 and 1727, respectively. Among the participants in cohort studies, 1367 cases of CHD were observed during a follow-up period ranging from 4.8 to 10.4 years, and 543 cases of stroke were observed during a follow-up period ranging from 4.8 to $10 \cdot 6$ years. All studies were conducted in Mediterranean countries. Only three studies were from Greece: a case-control study ${ }^{(26)}$ and two studies conducted within the EPIC (European Prospective Investigation into Cancer and Nutrition)-Greece cohort ${ }^{(25,27)}$. We included two Italian studies: a case-control study ${ }^{(11)}$ and the EPIC-Italy cohort $^{(28)}$. A cohort study assessing the incidence of stroke was conducted in France ${ }^{(16)}$. A casecontrol $^{(29)}$, a cohort ${ }^{(30)}$ and a randomised controlled trial ${ }^{(14)}$ were carried out in Spain.

Olive oil consumption was measured by validated FFQ in all studies; available intraclass correlation coefficients $v$. food records in published validation studies were $0 \cdot 71^{(14,29)}$, $0.50^{(11)}, 0.45$ for men and 0.70 for women ${ }^{(25-27)}, 0.48$ for men and 0.60 for women ${ }^{(28)}, 0.60$ for men and 0.67 for women ${ }^{(30)}$, respectively. Only the PREDIMED study ${ }^{(14)}$ used repeated measurements to update dietary information, using repeated FFQ during each year of follow-up to more accurately reflect the dietary intakes over the follow-up. In addition, the PREDIMED study was the single study that used biomarkers of virgin olive oil intake in a random subsample of participants. This assessment showed good compliance with the recommended consumption of virgin olive oil.

\section{Association between olive oil consumption and the risk of $\mathrm{CHD}$}

We included three case-control studies ${ }^{(11,26,29)}$, three cohort studies $^{(25,28,30)}$ with four reports and the PREDIMED trial ${ }^{(14)}$ in the present meta-analysis of olive oil consumption and the risk of CHD. We found no evidence for a significant association between olive oil consumption and the risk of CHD (Fig. 2). The random-effects model assessing CHD as an outcome showed a pooled OR of 0.73 (95\% CI 0.44 , $1 \cdot 21)$ in case-control studies and 0.96 (95\% CI $0.78,1.18)$ in cohort studies for a $25 \mathrm{~g}$ increase in olive oil consumption. The summary RR of CHD for an increase in olive oil consumption of $25 \mathrm{~g} / \mathrm{d}$ was 0.87 (95\% CI $0.72,1 \cdot 05 ; P=0 \cdot 14$; Table 4). We found evidence of heterogeneity among the studies $\left(P<0.001, I^{2}=77 \%\right.$; Fig. 2). The Egger regression test provided some evidence of publication bias $(P=0 \cdot 06)$. The trim and fill method ${ }^{(23)}$ did not add any study; therefore, the mean of the analysis remained unchanged. The exclusive consideration of cohort studies, either with the PREDIMED trial (RR 0.94, 95\% CI $0.78,1 \cdot 14$ ) or without it (RR 0.96, 95\% CI $0 \cdot 78,1 \cdot 18)$, also failed to find evidence of a significant inverse association between olive oil consumption and CHD.

\section{Association between olive oil consumption and the risk of stroke}

The present meta-analysis involved only three articles, two cohort studies ${ }^{(16,27)}$ and the PREDIMED trial ${ }^{(14)}$. All of them consistently reported significant inverse associations of a similar magnitude. For cohort studies assessing stroke, the combined RR of stroke for an increment of $25 \mathrm{~g}$ olive oil consumed per d was 0.76 (95\% CI 0.67, 0.86; $P<0.001$ ), with a negligible change after including the PREDIMED trial. No heterogeneity of effect estimates on RR was observed $\left(P=0 \cdot 44, I^{2}=0 \%\right)$. The Egger test for publication bias did not reach significance $(P=0 \cdot 11)$, although the number of studies was small.

\section{Association between olive oil consumption and the risk of $C V D$}

When all articles were combined, using either CHD or stroke as end-points, the combined RR of CVD for an increment of $25 \mathrm{~g}$ olive oil consumed per d was 0.82 (95\% CI 0.70, 0.96; $P=0 \cdot 01)$. Substantial heterogeneity of effect estimates on RR was observed $\left(P<0 \cdot 001, I^{2}=77 \%\right)$. The Egger test reached statistical significance $(P=0.06)$, and the funnel plot (Fig. 3; see online supplementary material) suggested that publication bias cannot be discarded.

No substantial differences were found when we separated the studies in three groups according to the inclusion of only women, only (or almost exclusively) men or an approximate $50 \%$ men and $50 \%$ women in the study (data not shown).

\section{Association between olive oil consumption and a dose-response trend in the risk of CHD and stroke}

When we used the method of Greenland \& O'Rourke ${ }^{(18)}$ to estimate the pooled RR associated with a $10 \mathrm{~g} / \mathrm{d}$ increment in olive oil consumption using all study quantiles, we needed to exclude the study by Kontogianni et al. ${ }^{(26)}$ because it did not provide specific information on separate categories of olive oil consumption and at least three categories are needed for the assessment of dose-response associations with this procedure. We found a RR of 0.94 (95\% CI 0.86, 1.03; random-effects model, $I^{2}=71 \%, P$ for heterogeneity=0.004) 
Table 1. Study design characteristics of nine epidemiological studies investigating incident myocardial infarction or stroke in relation to olive oil consumption

\begin{tabular}{|c|c|c|c|c|c|c|c|c|}
\hline Study & $\begin{array}{l}\text { Dietary assessment } \\
\text { method }\end{array}$ & $\begin{array}{l}\mathrm{FFQ} \\
\text { items }\end{array}$ & $\begin{array}{l}\text { Olive oil intake categorisation } \\
\text { (intervention in RCT) }\end{array}$ & $\begin{array}{l}\text { Validation } \\
\text { of nutrients }\end{array}$ & $\begin{array}{l}\text { Validation in the } \\
\text { study population }\end{array}$ & $\begin{array}{l}\text { Multiple } \\
\text { assessment } \\
\text { of diet }\end{array}$ & $\begin{array}{l}\text { Average duration of } \\
\text { follow-up (years) }\end{array}$ & Quality \\
\hline \multicolumn{9}{|l|}{ CHD event } \\
\hline \multicolumn{9}{|l|}{ Case-control } \\
\hline $\begin{array}{l}\text { Fernández-Jarne } \\
\text { et al. }(2002)^{(29)}\end{array}$ & FFQ (1 year) & 136 & Extreme quintiles & Validated & Yes & Baseline & NA & 8 \\
\hline $\begin{array}{l}\text { Bertuzzi et al. } \\
\quad(2002)^{(11)}\end{array}$ & FFQ (2 years) & 78 & Extreme quintiles & Validated & Yes & Baseline & NA & 5 \\
\hline $\begin{array}{l}\text { Kontogianni et al. } \\
(2007)^{(26)}\end{array}$ & $\begin{array}{l}\text { FFQ (time } \\
\text { frame NR) }\end{array}$ & NR & None, with other oils, exclusive & Not validated & - & Baseline & NA & 7 \\
\hline \multicolumn{9}{|l|}{ Cohort } \\
\hline \multirow[t]{2}{*}{$\begin{array}{l}\text { Bendinelli et al. } \\
\quad(2011)^{(28)}\end{array}$} & $\begin{array}{l}\text { FFQ (time } \\
\quad \text { frame NR) }\end{array}$ & 188 & Extreme quartiles & Validated & Yes & Baseline & $7 \cdot 85$ & 8 \\
\hline & & $\begin{array}{l}217 \\
140\end{array}$ & & & & & & \\
\hline $\begin{array}{l}\text { Buckland et al. } \\
\qquad(2012)^{(30)}\end{array}$ & $\begin{array}{l}\text { Open dietary } \\
\text { questionnaire } \\
\text { (1 year) }\end{array}$ & 662 & Extreme quartiles & Validated & Yes & Baseline & $10 \cdot 4$ & 8 \\
\hline Dilis et al. (2012) & $\mathrm{FFQ}$ & 200 & Per increment increase in consumption & Validated & Yes & Baseline & 10 & 9 \\
\hline \multicolumn{9}{|l|}{ Stroke } \\
\hline \multicolumn{9}{|l|}{ Cohort } \\
\hline Samieri et al. (2011) $)^{(16)}$ & Brief FFQ & 12 & No, moderate, intense use & None & - & Baseline & $5 \cdot 25$ & 8 \\
\hline Misirli et al. (2012) (27) & FFQ & 150 & Per increment increase in consumption & Validated & Yes & Baseline & $10 \cdot 6$ & 9 \\
\hline \multicolumn{9}{|l|}{$\begin{array}{l}\text { RCT: both myocardial } \\
\text { infarction and stroke }\end{array}$} \\
\hline PREDIMED $(2013)^{(14)}$ & $\begin{array}{l}\text { FFQ (annually } \\
\text { repeated) }\end{array}$ & 137 & $\begin{array}{l}\text { Intervention: Mediterranean diet } \\
\text { supplemented with extra-virgin olive oil } \\
\text { v. a control diet (advice to reduce dietary fat) }\end{array}$ & Validated & Yes & Yes & $4 \cdot 8$ & \\
\hline
\end{tabular}

$\mathrm{RCT}$, randomised controlled trial; NA, not applicable; NR, not reported. 


\section{N British Journal of Nutrition}

Table 2. Characteristics of nine epidemiological studies investigating incident myocardial infarction or stroke in relation to olive oil intake (Mean or median values and ranges)

\begin{tabular}{|c|c|c|c|c|c|c|c|c|c|}
\hline \multirow[b]{2}{*}{ Study } & \multirow[b]{2}{*}{$\begin{array}{l}\text { No. of } \\
\text { participants }\end{array}$} & \multicolumn{2}{|c|}{ Age (years) } & \multirow[b]{2}{*}{$\begin{array}{l}\text { Country of } \\
\text { residence }\end{array}$} & \multirow[b]{2}{*}{$\begin{array}{l}\text { Time of data } \\
\text { acquisition }\end{array}$} & \multirow[b]{2}{*}{$\begin{array}{l}\text { No. of } \\
\text { centres }\end{array}$} & \multirow[b]{2}{*}{$\begin{array}{l}\text { Smokers, among } \\
\text { cases }(\%)\end{array}$} & \multirow[b]{2}{*}{ Disease outcome } & \multirow[b]{2}{*}{ Method of diagnosis } \\
\hline & & $\begin{array}{l}\text { Mean or } \\
\text { median }\end{array}$ & Range & & & & & & \\
\hline \\
\hline & & & & & \multicolumn{4}{|c|}{ Case-control } & \\
\hline $\begin{array}{l}\text { Fernández-Jarne } \\
\text { et al. }(2002)^{(29)}\end{array}$ & 342 & $\begin{array}{l}\text { Cases: } 61 \cdot 7 \\
\quad \text { controls: } 61 \cdot 4\end{array}$ & & Spain & 1999-2001 & 1 & Current: 40; ex: 29 & Survivors of AMI & MONICA project criteria* \\
\hline $\begin{array}{l}\text { Bertuzzi } \\
\text { et al. (2002) }\end{array}$ & 985 & 61 & $25-79$ & Italy & $1995-9$ & 478 & $45 \cdot 6$ & Non-fatal AMI & $\begin{array}{l}\text { First episode of non-fatal AMI, defined according to } \\
\text { the WHO criteria }\end{array}$ \\
\hline $\begin{array}{l}\text { Kontogianni } \\
\text { et al. }(2007)^{(26)}\end{array}$ & 1926 & $\begin{array}{l}\text { Cases: } 60.7 \\
\quad \text { controls: } 58 \cdot 7\end{array}$ & & Greece & $2000-2$ & $\begin{array}{l}\text { NR } \\
\text { (multi-centre) }\end{array}$ & $\begin{array}{l}63 \text { (no use of olive oil); } \\
67 \text { (exclusive use); } \\
70 \text { (+ other oils) }\end{array}$ & $\begin{array}{l}\text { First, AMI or } \\
\text { unstable angina }\end{array}$ & $\begin{array}{l}\text { ECG, symptoms, enzyme elevations or unstable } \\
\text { angina (class III Braunwald classification) }\end{array}$ \\
\hline \multicolumn{10}{|c|}{ 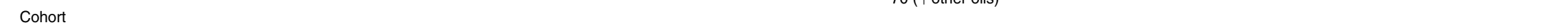 } \\
\hline $\begin{array}{l}\text { Bendinelli } \\
\quad \text { et al. }(2011)^{(28)}\end{array}$ & 29689 & 50 & $35-74$ & Italy & $\begin{array}{l}1993-8 \\
\text { (recruitment) }\end{array}$ & 5 & NR & $\begin{array}{l}\text { AMI (fatal or } \\
\text { non-fatal), } \\
\text { revascularisation } \\
\text { or both }\end{array}$ & $\begin{array}{l}\text { EPIC lifestyle questionnaire + medical record } \\
\text { linkage }\end{array}$ \\
\hline $\begin{array}{l}\text { Buckland } \\
\text { et al. }(2012)^{(30)}\end{array}$ & 40142 & 49 & $29-69$ & Spain & $\begin{array}{l}1992-6 \\
\text { (recruitment) }\end{array}$ & 5 & 69.2 & $\begin{array}{l}\text { Non-fatal and fatal } \\
\text { CHD events }\end{array}$ & $\begin{array}{l}\text { Self-reported CHD events and record linkage with } \\
\text { hospital discharge databases, population-based } \\
\text { AMI registries, and regional and national mortality } \\
\text { registries, validated by trained nurses/physicians } \\
\text { according to the } 2003 \text { AHA Statement and } \\
\text { MONICA criteria }\end{array}$ \\
\hline Dilis et al. $(2012)^{(25)}$ & 23929 & NR & $20-86$ & Greece & $\begin{array}{l}\text { 1994-9 } \\
\text { (recruitment) }\end{array}$ & $\begin{array}{l}\text { NR } \\
\text { (nationwide) }\end{array}$ & Current: 30; ex: 22.6 & $\begin{array}{l}\text { First CHD event } \\
\text { (AMI, angina and } \\
\text { CHD other than } \\
\text { angina or AMI) }\end{array}$ & $\begin{array}{l}\text { Self-reported CHD events during the follow-up, } \\
\text { confirmed through hospital discharge data, } \\
\text { medical records or death certificates, and } \\
\text { classified according to the 10th revision of the ICD }\end{array}$ \\
\hline \multicolumn{10}{|r|}{ 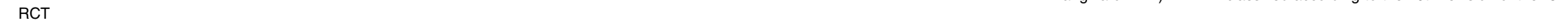 } \\
\hline $\begin{array}{r}\text { PREDIMED } \\
(2013)^{(14)}\end{array}$ & 7447 & 67 & $55-80$ & Spain & $2003-10$ & 11 & $\begin{array}{l}\text { Current: } 13 \cdot 9 \\
\quad \text { ex: } 24 \cdot 3 \\
\text { (at baseline) }\end{array}$ & AMI & $\begin{array}{l}\text { Information from four sources: repeated contacts } \\
\text { with participants, family physicians, annual review } \\
\text { of medical records and consultation of the } \\
\text { National Death Index. An independent blinded } \\
\text { adjudication committee assigned end-points using } \\
\text { standardised criteria }\end{array}$ \\
\hline \multicolumn{10}{|r|}{ 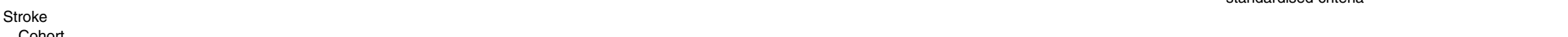 } \\
\hline $\begin{array}{l}\text { Samieri } \\
\text { et al. }(2011)^{(16)}\end{array}$ & 7625 & 73.8 & & France & 1999-ongoing & 3 & NR & Stroke & $\begin{array}{l}\text { Information from interviews at 2, } 4 \text { and } 6 \text { years and } \\
\text { medical records being reviewed in those screened } \\
\text { positive, using the WHO diagnostic criteria for } \\
\text { stroke }\end{array}$ \\
\hline $\begin{array}{l}\text { Misirli } \\
\text { et al. (2012) }\end{array}$ & 23601 & $\begin{array}{l}<55=58 \% \\
\quad 55-64=23 \% \\
\geq 65=19 \%\end{array}$ & & Greece & $\begin{array}{l}\text { 1994-9 } \\
\quad \text { (recruitment) }\end{array}$ & $\begin{array}{l}\text { NR } \\
\text { (nationwide) }\end{array}$ & Current: 19; ex: 23 & Stroke & $\begin{array}{l}\text { Information from interviews and verification through } \\
\text { pathology reports, medical records, discharge } \\
\text { diagnoses or death certificates at the local death } \\
\text { registries }\end{array}$ \\
\hline \multicolumn{10}{|r|}{ 皮 } \\
\hline $\begin{array}{r}\text { PREDIMED } \\
(2013)^{(14)}\end{array}$ & 7447 & 67 & $55-80$ & Spain & $2003-10$ & 11 & $\begin{array}{l}\text { Current: } 13 \cdot 9 \\
\quad \text { ex: } 24 \cdot 3 \\
\text { (at baseline) }\end{array}$ & Stroke & $\begin{array}{l}\text { Information from four sources: repeated contacts } \\
\text { with participants, family physicians, annual review } \\
\text { of medical records and consultation of the } \\
\text { National Death Index. An independent blinded } \\
\text { adjudication committee assigned end-points using } \\
\text { standardised criteria }\end{array}$ \\
\hline
\end{tabular}

AMI, acute myocardial infarction; MONICA, multinational MONItoring of trends and determinants in CArdiovascular disease; NR, not reported; ECG, electrocardiogram; EPIC, European Prospective Investigation into Cancer and Nutrition; AHA, American Heart Association; ICD, International Classification of Diseases; RCT, randomised controlled trial.

*Two or more ECG showing specific changes, ECG showing probable changes plus abnormal cardiac enzymes, or typical symptoms plus abnormal enzymes. 
Table 3. Relative risk (RR) estimates for the association of olive oil intake and incident myocardial infarction or stroke in nine epidemiological studies

(Relative risks and $95 \%$ confidence intervals)

\begin{tabular}{|c|c|c|c|c|c|c|c|c|c|c|c|c|}
\hline Study & Design & $\begin{array}{l}\text { Sex, } \\
\text { males } \\
(\%)\end{array}$ & $\begin{array}{l}\text { No. of } \\
\text { cases }\end{array}$ & $\begin{array}{l}\text { No. of } \\
\text { controls }\end{array}$ & $\begin{array}{l}\text { No. of } \\
\text { non-fatal } \\
\text { events }\end{array}$ & $\begin{array}{l}\text { No. of } \\
\text { fatal } \\
\text { events }\end{array}$ & $\begin{array}{l}\text { Median or } \\
\text { mean olive } \\
\text { oil intake }\end{array}$ & Covariates in the fully adjusted model & $\begin{array}{l}\text { Original } \\
\text { reported } \\
\text { RR }\end{array}$ & $95 \% \mathrm{Cl}$ & $\begin{array}{l}\text { RR for } \\
+25 \mathrm{~g} / \mathrm{d} \text { of } \\
\text { consumption }\end{array}$ & $95 \% \mathrm{Cl}$ \\
\hline \multicolumn{13}{|l|}{ CHD events } \\
\hline $\begin{array}{l}\text { Fernández-Jarne } \\
\text { et al. }(2002)^{(29)}\end{array}$ & $\begin{array}{l}\text { Case- } \\
\text { control }\end{array}$ & 81 & 171 & 171 & 171 & 0 & $24.9 \mathrm{~g} / \mathrm{d}$ & $\begin{array}{l}\text { Age, sex, smoking status, BMI, high blood pressure, } \\
\text { high blood cholesterol, diabetes, leisure-time } \\
\text { physical activity level (MET-h/week), marital } \\
\text { status, occupation and study level, percentage of } \\
\text { energy derived from saturated fat, trans-fat, total } \\
\text { fibre consumption, folic acid intake, vitamin C } \\
\text { intake, glycaemic load and ethanol intake }\end{array}$ & OR 0.18 & $0.05,0.63$ & 0.40 & $0.20,0.78$ \\
\hline $\begin{array}{l}\text { Bertuzzi } \\
\text { et al. } \\
(2002)^{(11)}\end{array}$ & $\begin{array}{l}\text { Case- } \\
\text { control }\end{array}$ & $\begin{array}{l}75 \cdot 6 \\
\quad \text { cases; } \\
62 \cdot 1 \\
\text { controls }\end{array}$ & 507 & 478 & 507 & 0 & $35.6 \mathrm{~g} / \mathrm{d}$ & $\begin{array}{l}\text { Age, sex, educational status, BMI, cholesterol, } \\
\text { smoking status, intakes of coffee, alcohol, energy, } \\
\text { butter, margarine and seed oil, physical activity } \\
\text { level, diabetes, hyperlypidaemia, hypertension }\end{array}$ & OR 1.48 & $0.86,2.55$ & $1 \cdot 14$ & $0.95,1 \cdot 37$ \\
\hline $\begin{array}{l}\text { Kontogianni } \\
\text { et al. (2007) } \\
\text { (CARDIO2000 } \\
\text { study) } 26)\end{array}$ & $\begin{array}{l}\text { Case- } \\
\text { control }\end{array}$ & $\begin{array}{l}81.7 \\
\quad \text { cases; } \\
71 \cdot 3 \\
\text { controls }\end{array}$ & 848 & 1078 & 848 & 0 & NR & $\begin{array}{l}\text { Age, sex, BMI, smoking status, physical activity } \\
\text { level, educational status, presence of family } \\
\text { history of CHD, hypertension, hyper- } \\
\text { cholesterolaemia, diabetes }\end{array}$ & OR 0.53 & $0.34,0.71$ & 0.67 & $0.51,0.81$ \\
\hline $\begin{array}{l}\text { Bendinelli } \\
\text { et al. (2011) } \\
\text { (EPICORStudy) }^{(28)}\end{array}$ & Cohort & 0 & 144 & & $\begin{array}{l}104 \text { AMl; } 27 \\
\text { revascularisation }\end{array}$ & 13 & $24.7 \mathrm{~g} / \mathrm{d}$ & $\begin{array}{l}\text { Age (as time scale), educational status, smoking } \\
\text { status, alcohol consumption, bodd height, body } \\
\text { weight, waist circumference, daily non-alcoholic } \\
\text { energy intake, hypertension, menopausal status, } \\
\text { physical activity level, consumption of meat, fruit } \\
\text { and vegetables }\end{array}$ & HR 0.56 & $0.31,0.99$ & 0.45 & $0.20,0.99$ \\
\hline $\begin{array}{l}\text { Buckland } \\
\text { et al. (2012) } \\
\text { (EPIC-Spain) }^{(30)}\end{array}$ & Cohort & 38 & 587 & & NR & NR & $\begin{array}{l}10 \mathrm{~g} / \mathrm{d} \mathrm{per} \\
8368 \mathrm{~kJ} \\
(2000 \mathrm{kcal})\end{array}$ & $\begin{array}{l}\text { Educational status, BMI, waist circumference, } \\
\text { physical activity level, smoking status, alcohol } \\
\text { consumption, energy intake excluding alcohol, } \\
\text { hyperlipidaemia, hypertension, diabetes, } \\
\text { Mediterranean diet score (excluding olive oil and } \\
\text { alcohol), excluding participants with improbable } \\
\text { dietary values according to the Goldberg criteria }\end{array}$ & HR 0.93 & $0.87,1 \cdot 0$ & 0.83 & $0.71,1.0$ \\
\hline $\begin{array}{l}\text { Dilis et al. } \\
\text { (2012) } \\
\text { (EPIC- }\end{array}$ & Cohort & $40 \cdot 7$ & 636 & & 396 & 240 & $\begin{array}{l}\text { Men: } 53 \mathrm{~g} / \mathrm{d} ; \\
\text { womem: } \\
45 \mathrm{~g} / \mathrm{d}\end{array}$ & $\begin{array}{l}\text { Age, BMI, height, physical activity level, years of } \\
\text { schooling, energy intake, alcohol consumption, } \\
\text { smoking status and arterial BP }\end{array}$ & $\begin{array}{l}\text { Men: } \\
\text { HR } 1.09 \\
\text { Women }\end{array}$ & $\begin{array}{l}0.97,1.23 \\
0.90-1.34\end{array}$ & $\begin{array}{l}\text { Men: } \\
\quad 1.09 \\
\text { Women }\end{array}$ & $\begin{array}{l}0.97,1.24 \\
0.891 .39\end{array}$ \\
\hline $\begin{array}{l}\text { Greece })^{(25)} \\
\text { PREDIMED }\end{array}$ & & & & & & & & & HR $1 \cdot 10$ & $0.90,1.34$ & $\begin{array}{c}\text { Women: } \\
1.11\end{array}$ & $0.89,1.39$ \\
\hline $\begin{array}{l}\text { PREDIMED } \\
(2013)^{(14)}\end{array}$ & RCT & 43 & $\begin{array}{l}\text { Control: } 38 ; \\
\quad \text { intervention: } \\
37\end{array}$ & & - & - & $\begin{array}{l}\text { Control: } 2 \cdot 6 \mathrm{~g} / \mathrm{d} \text {; } \\
\text { intervention: } \\
29 \cdot 3 \mathrm{~g} / \mathrm{d}\end{array}$ & $\begin{array}{l}\text { BMI, waistheight ratio, hypertension, dyslipidaemia } \\
\text { and diabetes at baseline }\end{array}$ & HR 0.8 & $0.51,1.26$ & 0.81 & $0.53,1.24$ \\
\hline $\begin{array}{l}\text { Stroke } \\
\text { Samieri et al. } \\
(2011) \\
(\text { Three-City } \\
\text { Study) })^{(16)}\end{array}$ & Cohort & 37.7 & 148 & & NR & NR & NR & $\begin{array}{l}\text { Age, sex, educational status, centre, consumption of } \\
\text { fish, meat, pulses, raw vegetables, raw fruits, } \\
\text { cooked fruits and vegetables, cereals, regular use } \\
\text { of } n \text {-3-rich oils, } n-6-\text {-rich oils, butter, goose or duck } \\
\text { fat, alcohol consumption, physical activity level, } \\
\text { systolic blood pressure, diabetes, antihyperten- } \\
\text { sive therapy, smoking status, history of CVD, AF, } \\
\text { BMI, triacylglycerolaemia and hyper- } \\
\text { cholesterolaemia }\end{array}$ & HR 0.59 & $0.37,0.94$ & 0.59 & $0.37,0.94$ \\
\hline $\begin{array}{l}\text { Misirli et al. } \\
(2012) \\
\text { (EPIC- } \\
\text { Greece) })^{(27)}\end{array}$ & Cohort & 40.7 & 395 & & 199 & 196 & $48.1 \mathrm{~g} / \mathrm{d}$ & $\begin{array}{l}\text { Age, sex, educational status, smoking status, BMI, } \\
\text { physical activity level (MET), hypertension, } \\
\text { diabetes, total energy intake }\end{array}$ & $\begin{array}{l}\text { Incidence } \\
\text { HR } 0.80 \\
\text { Mortality } \\
\text { HR } 0.89\end{array}$ & $\begin{array}{l}0.70-0.90 \\
0.73-1.08\end{array}$ & $\begin{array}{l}\text { Incidence } \\
0.78 \\
\text { Mortality } \\
0.88\end{array}$ & $\begin{array}{l}0.68-0.89 \\
0.71-1.08\end{array}$ \\
\hline $\begin{array}{l}\text { PREDIMED } \\
(2013)^{(14)}\end{array}$ & $\mathrm{RCT}$ & 43 & $\begin{array}{l}\text { Control: } 58 \text {; } \\
\text { intervention: } \\
49\end{array}$ & & - & - & $\begin{array}{l}\text { Control: } \\
2 \cdot 6 \mathrm{~g} / \mathrm{d} ; \\
\text { intervention: } \\
29 \cdot 3 \mathrm{~g} / \mathrm{d}\end{array}$ & $\begin{array}{l}\text { BMI, waist:height ratio, } \\
\text { hypertension, } \\
\text { dyslipidaemia and } \\
\text { diabetes at baseline }\end{array}$ & HR 0.67 & $0.46-0.98$ & 0.69 & $0.48-0.98$ \\
\hline
\end{tabular}

MET, metabolic equivalents; AMI, acute myocardial infarction; NR, not reported; HR, hazard ratio; EPIC, European Prospective Investigation into Cancer and Nutrition; BP, blood pressure; RCT, randomised controlled trial; AF, atrial fibrillation. 


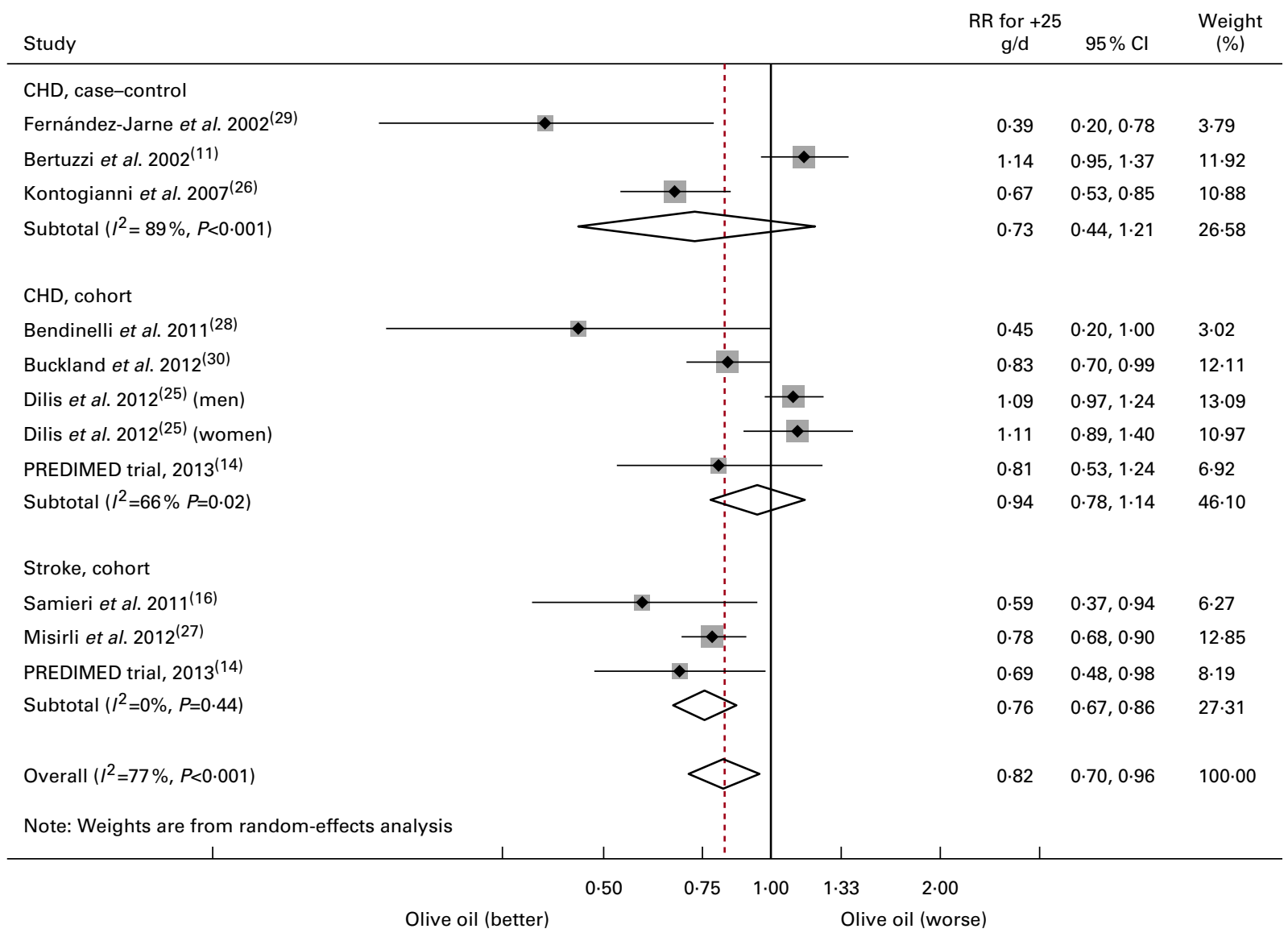

Fig. 2. Relative risks (RR) and $95 \% \mathrm{Cl}$ for fully adjusted random-effects models examining the associations between olive oil consumption in relation to the incidence of CHD and stroke in the meta-analysis of nine studies (three case-control, five cohort studies and one randomised controlled trial). (A colour version of this figure can be found online at http://www.journals.cambridge.org/bjn).

for CHD and a RR of 0.90 (95\% CI 0.86, 0.95; fixed-effects model; $I^{2}=0 \%, \quad P$ for heterogeneity $=0.61$ ) for stroke. Table 5 shows study quality assessment.

We also conducted a sensitivity analysis to ascertain whether significant outcomes were related to any one particular study, by sequentially excluding each of them, one at a time. As shown in Table 6 (see also online supplementary material), the results were similar when each particular study was excluded.

\section{Discussion}

The present meta-analysis identified no significant association between olive oil consumption and the risk of $\mathrm{CHD}$, but

Table 4. Relative risks (RR) of $\mathrm{CHD}$ and stroke according to design and outcome*

(Relative risks and $95 \%$ confidence intervals)

\begin{tabular}{|c|c|c|c|c|c|c|c|}
\hline & \multirow[b]{2}{*}{ No. of studies } & \multicolumn{3}{|c|}{ Effect } & \multicolumn{3}{|c|}{ Heterogeneity } \\
\hline & & $\mathrm{RR}$ & $95 \% \mathrm{Cl}$ & $P$ & $Q$ & $P$ for heterogeneity & $I^{2}(\%)$ \\
\hline \multicolumn{8}{|l|}{$\mathrm{CHD}$} \\
\hline All, random & 7 & 0.87 & $0.71,1.07$ & 0.19 & 30.9 & $<0.001$ & $80 \cdot 6$ \\
\hline All, fixed & 7 & 0.97 & $0.90,1.05$ & 0.47 & & & \\
\hline All, +PREDIMED, random & 8 & 0.87 & $0.72,1.05$ & 0.14 & 31.5 & 0.001 & $77 \cdot 8$ \\
\hline Case-control, random & 3 & 0.73 & $0.44,1.21$ & 0.22 & $18 \cdot 2$ & $<0.001$ & $89 \cdot 0$ \\
\hline Cohort, random & 4 & 0.96 & $0.78,1.18$ & 0.69 & $10 \cdot 9$ & 0.01 & $72 \cdot 5$ \\
\hline Cohort, fixed & 4 & 1.01 & $0.92,1.10$ & 0.89 & & & \\
\hline Cohort, + PREDIMED, random & 5 & 0.94 & $0.78,1.14$ & 0.52 & 11.8 & 0.02 & $66 \cdot 2$ \\
\hline \multicolumn{8}{|l|}{ Stroke } \\
\hline Cohort, random & 2 & 0.74 & $0.60,0.92$ & 0.007 & 1.32 & 0.25 & 24.4 \\
\hline Cohort, fixed & 2 & 0.77 & $0.67,0.87$ & $<0.001$ & & & \\
\hline Cohort, + PREDIMED, random & 3 & 0.76 & $0.67,0.86$ & $<0.001$ & 1.65 & 0.44 & 0.0 \\
\hline
\end{tabular}

PREDIMED, PREvención con Dleta MEDiterránea (Prevention with Mediterranean Diet).

${ }^{*}$ All cardiovascular end-points combined: $P$ (Egger test) for publication bias - all, $P=0.06 ; \mathrm{CHD}, P=0.07 ;$ stroke, $P=0.11$. 


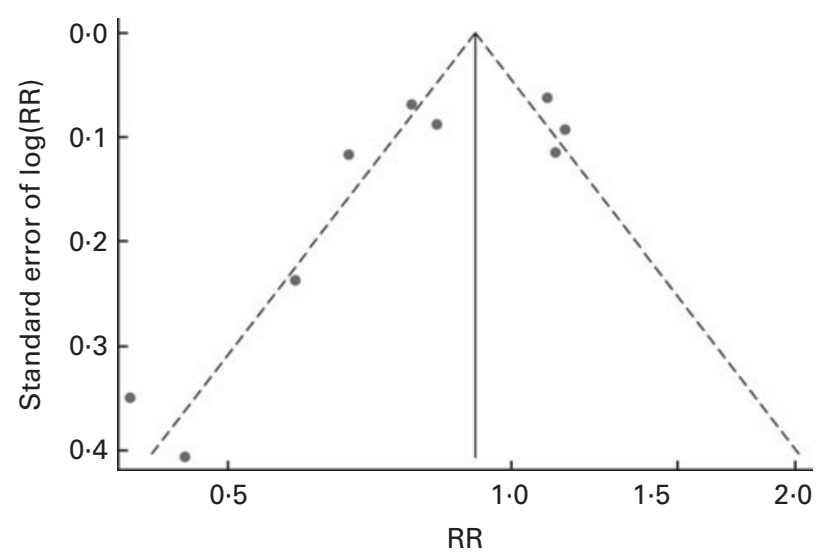

Fig. 3. Funnel plot of studies (three case-control, five cohort studies and one randomised controlled trial) investigating olive oil consumption in relation to the incidence of CVD (CHD and stroke; see online supplementary material). ----, Pseudo $95 \% \mathrm{Cl}$. RR, relative risk.

a significantly reduced risk of stroke associated with the consumption of olive oil. Substantial heterogeneity among studies was observed for CHD, but not for stroke. Combined results of observational epidemiological studies for both CHD and stroke are in agreement with the recent findings of the PREDIMED randomised controlled trial. The PREDIMED trial reported that participants who were allocated to receive a quarterly allotment of 15 litres of virgin olive oil obtained a significant $30 \%$ reduction in the primary end-point of the trial, which was a composite of myocardial infarction or stroke or death from cardiovascular causes ${ }^{(14)}$. However, with respect to the components of the primary end-point, only the comparisons of stroke risk reached statistical significance in the PREDIMED trial, and no significant inverse associations were obtained for myocardial infarction or death from cardiovascular causes. It should be kept in mind, as a limitation for inference, that publication bias or smallstudy effects were present. Regarding publication bias, although the Egger test detected a significant influence of small studies, the trim and fill method did not add any further study; nevertheless, it should be emphasised that this method lacks statistical power when the number of primary studies is less than ten.

The inverse association between olive oil consumption and the risk of stroke was fairly consistent, but the scarcity of studies on this outcome should be acknowledged. The absence of significant results for CHD should not be viewed as surprising or disappointing. It is true that olive oil is the hallmark of the Mediterranean dietary pattern and that this dietary pattern is able to obtain a sizable reduction in cardiovascular risk $^{(31,32)}$. This apparent paradox can be explained because of several reasons: (1) any single dietary factor is unlikely to have a large effect on the risk of a multifactorial disease, such as CHD; (2) the appropriate exposure to be assessed should probably be virgin olive oil instead of all kinds of olive oil mixed together; (3) misclassification bias (with a higher propensity to shift estimates towards the null than away from the null) is likely to be present in all included studies because of the inherent measurement errors present in the tools used to evaluate olive oil consumption; (4) overadjustment for intermediate mechanisms by which olive oil can reduce the risk of CHD would also attenuate the potential association between olive oil consumption and the risk of CHD. It is recognised that in an attempt to estimate the total effect of an exposure on some outcome, control for intermediate factors in the causal chain (i.e. overadjustment) will generally bias estimates of the total effect of the exposure on the outcome ${ }^{(33)}$. One of the mechanisms by which olive oil is suggested to work is through its effects on lipid profiles. Only two of the studies ${ }^{(25,28)}$ reported the association between olive oil intake and the risk of CHD without adjusting for serum lipid concentrations, and they found a positive ${ }^{(28)}$ and a negative ${ }^{(25)}$ association. In any case, it is noteworthy to remark that an intermediate link, such as lipid concentrations, in the causal chain should not be treated as a confounder.

In a previous assessment of Mediterranean diet and mortality in the EPIC-Greece cohort $^{(34)}$, olive oil was associated with only a small and non-significant reduction in mortality, whereas the inverse association between mortality and the ratio of monounsaturated fats:saturated fats was stronger and statistically significant. This finding also contributes to the view that the overall pattern has a beneficial effect beyond that of olive oil.

In addition, other plant-based sources of MUFA such as nuts and other vegetable oils can have a similar effect than olive oil on blood lipids, inflammatory pathways and oxidative stress. There is a possibility that the effect of minor polyphenolic components of olive oil, which are present in the virgin variety of olive oil but not in the ordinary refined variety, are able to make a large difference in the potential protection that olive oil consumption can afford against the risk of CHD. In the PREDIMED trial, the intervention provided only virgin olive oil. Differences in the intake of both varieties (virgin olive oil $v$. ordinary refined variety, which is low in polyphenols) were not taken into account in most observational studies included in the present meta-analysis, with only one exception being the study by Buckland et al. ${ }^{(30)}$ that reported a stronger protection among consumers of virgin olive oil.

Table 5. Quality assessment of studies according to the NewcastleOttawa Scale ${ }^{(17)}$

\begin{tabular}{|c|c|c|c|c|}
\hline & Selection & Comparability & $\begin{array}{c}\text { Exposure/ } \\
\text { outcome }\end{array}$ & Total \\
\hline $\begin{array}{l}\text { Fernández-Jarne } \\
\text { et al. }(2002)^{(29)}\end{array}$ & 3 & 2 & 3 & 8 \\
\hline $\begin{array}{l}\text { Bertuzzi } \\
\quad \text { et al. }(2002)^{(11)}\end{array}$ & 1 & 2 & 2 & 5 \\
\hline $\begin{array}{l}\text { Kontogianni } \\
\text { et al. }(2007)^{(26)}\end{array}$ & 3 & 2 & 2 & 7 \\
\hline $\begin{array}{l}\text { Bendinelli } \\
\text { et al. }(2011)^{(28)}\end{array}$ & 4 & 2 & 2 & 8 \\
\hline $\begin{array}{l}\text { Buckland } \\
\text { et al. }(2012)^{(30)}\end{array}$ & 4 & 2 & 2 & 8 \\
\hline $\begin{array}{l}\text { Dilis et al. } \\
\quad(2012)^{(25)}\end{array}$ & 4 & 2 & 3 & 9 \\
\hline $\begin{array}{c}\text { Samieri et al. } \\
(2011)^{(16)}\end{array}$ & 4 & 2 & 2 & 8 \\
\hline $\begin{array}{l}\text { Misirli et al. } \\
(2012)^{(27)}\end{array}$ & 4 & 2 & 3 & 9 \\
\hline
\end{tabular}


Table 6. Sensitivity analysis of relative risks (RR) for each study being removed at a time (Relative risks and $95 \%$ confidence intervals)

\begin{tabular}{|c|c|c|c|c|c|c|c|c|}
\hline \multirow[b]{3}{*}{ Study excluded } & \multicolumn{4}{|c|}{$\mathrm{CHD}$} & \multicolumn{4}{|c|}{ Stroke } \\
\hline & \multicolumn{2}{|c|}{ Fixed effects } & \multicolumn{2}{|c|}{ Random effects } & \multicolumn{2}{|c|}{ Fixed effects } & \multicolumn{2}{|c|}{ Random effects } \\
\hline & $\mathrm{RR}$ & $95 \% \mathrm{Cl}$ & $\mathrm{RR}$ & $95 \% \mathrm{Cl}$ & $\mathrm{RR}$ & $95 \% \mathrm{Cl}$ & $\mathrm{RR}$ & $95 \% \mathrm{Cl}$ \\
\hline PREDIMED $(2013)^{(14)}$ & 0.97 & $0.90,1.05$ & 0.87 & $0.71,1.07$ & 0.77 & $0.67,0.88$ & 0.75 & $0.60,0.92$ \\
\hline Misirli et al. (2012) & & & & & 0.65 & $0.49,0.86$ & 0.65 & $0.49,0.86$ \\
\hline Samieri et al. $(2011)^{(16)}$ & & & & & 0.77 & $0.68,0.88$ & 0.77 & $0.68,0.88$ \\
\hline Dilis et al. (2012) ${ }^{(25)}$ (women) & 0.95 & $0.88,1.03$ & 0.83 & $0.77,1.02$ & & & & \\
\hline Dilis et al. $(2012)^{(25)}$ (men) & 0.90 & $0.82,0.99$ & 0.82 & $0.65,1.02$ & & & & \\
\hline Buckland et al. $(2012)^{(30)}$ & 1.00 & $0.92,1.09$ & 0.87 & $0.70,1.08$ & & & & \\
\hline Bendinelli et al. $(2011)^{(28)}$ & 0.97 & $0.90,1.05$ & 0.90 & $0.75,1.08$ & & & & \\
\hline Kontogianni et al. (2007) $)^{(26)}$ & 1.01 & $0.93,1.09$ & 0.93 & $0.77,1.11$ & & & & \\
\hline Bertuzzi et al. (2002) $)^{(11)}$ & 0.94 & $0.86,1.02$ & 0.82 & $0.66,1.02$ & & & & \\
\hline Fernández-Jarne et al. (2002) & 0.98 & $0.91,1.05$ & 0.92 & $0.77,1.09$ & & & & \\
\hline
\end{tabular}

There is a need for further studies that fully take into account these differences given the available evidence to support a beneficial effect of these polyphenols on coronary risk factors ${ }^{(35-38)}$.

An important point is whether persons consuming more olive oil may reduce their intake of energy from other macronutrients to maintain an energy balance. This would be pertinent to stroke which is often considered to be elevated among individuals having higher intakes of carbohydrate ${ }^{(39)}$. However, the results of all the studies included in the present meta-analysis were adjusted for total energy intake, which may reduce this possible source of confounding. Another important point to consider is that a lower risk of stroke may not due only to olive oil intake, but also due to a lower carbohydrate or lower glycaemic load, which is another hallmark of the Mediterranean diet. However, with the information available in the primary studies included in the present meta-analysis, it is not possible to solve this question, and future studies specifically designed to address this specific issue are warranted.

More importantly, emphasis is needed to assert that the available observational evidence on olive oil consumption and hard clinical events of CVD is scarce in spite of many published reviews and many reports of mechanistic studies using only intermediate end-points as the outcome. The small number of studies available for inclusion in the present meta-analysis forced us to combine different study designs, each with distinct limitations (e.g. residual confounding in prospective cohort studies and recall bias in retrospective case-control studies, while randomised controlled trials should in principle be free of both biases). The limitation derived from the few number of available studies also applies to the $P$ value for Cochran's $Q$ test of heterogeneity, which would be underpowered, and does not accurately quantify heterogeneity. Unfortunately, the scarce number of studies did not allow us to explore detailed assessments to explain heterogeneity, such as subgroup, stratified analysis or metaregression. The current recommendation is that at least ten studies are needed to perform meta-regression analysis ${ }^{(40)}$. Although this is a general recommendation and fewer studies are possible when goodness of fit is appropriate or when it becomes a practical necessity or in hypothesis development, perhaps, and more importantly, underlying assumptions of heterogeneous methods may limit the reliability of metaregression analysis when the number of studies is scarce.

Nevertheless, some points can be discussed regarding possible sources of heterogeneity observed in the present meta-analysis. There are two studies in the forest plot (Fig. 2) that deviate from the general result, indicating a nonfavourable effect of olive oil on the incidence of CHD events: one is a case-control study by Bertuzzi et al. ${ }^{(11)}$ and another is a cohort study by Dilis et $a l^{(25)}$. The first study ${ }^{(11)}$ did not have a matched design compared with the other case-control study ${ }^{(29)}$ included in the meta-analysis. The case-control study using a matched design reported instead an inverse association ${ }^{(29)}$. More importantly, another possible source of heterogeneity might be related to the case definition and eligibility criteria; in the study by Bertuzzi et al. ${ }^{(11)}$, patients with a previous history of CVD were not explicitly excluded. It is possible that subjects who know or suspect that they had previous manifestations of CHD may be motivated to increase their consumption of olive oil (i.e. a reverse causality bias might account for a direct association between olive oil consumption and the risk of CHD if subjects with a previous history of CVD are not excluded).

Regarding the cohort study by Dilis et al. ${ }^{(25)}$, a possible source of heterogeneity may lie on the fact that the mean olive oil intake at baseline, both in men and women, was very high (53 and $45 \mathrm{~g} / \mathrm{d}$, respectively), in comparison with other cohort studies ${ }^{(28,30)}(24.7$ and $10 \mathrm{~g} / \mathrm{d}$ per $8368 \mathrm{~kJ}$ ( $2000 \mathrm{kcal})$, respectively) and with the intervention arm of the PREDIMED trial ${ }^{(14)}(29 \cdot 3 \mathrm{~g} / \mathrm{d})$. High olive oil consumption at baseline, characteristic of the population from Greece, may have attenuated the ability to identify an association between olive oil intake and the risk of CHD, because even subjects in the lower categories may consume a sufficient amount.

The quality of some case-control studies, in accordance with the modified $\operatorname{NOS}^{(17)}$, was not high (Table 5; see online supplementary material). The publication of the PREDIMED randomised controlled trial is supportive and demonstrative of the protection afforded by virgin olive oil against a combined cardiovascular end-point and also against the stroke component of this combined end-point. However, a randomised controlled trial also has inherent limitations including 
limited external validity of its results, the lack of ability to ascertain a dose-response trend and the constraint for inferences to be made only to the limits of the attained contrast (really observed differences) between the intervention and the control groups. No conclusion can be inferred beyond the limits of the observed contrast in randomised trials. In this sense, the intervention group of the PREDIMED trial exhibited good compliance with the intended goals of virgin olive oil consumption, but participants allocated to the control group were fairly similar to those of the intervention group in many aspects of their diets, and only small between-group differences in the diets during the trial were observed.

In conclusion, the overall evidence supports a protection of olive oil consumption against hard cardiovascular end-points, and specifically against stroke. However, the evidence is scarce and no significant protection can be afforded specifically against CHD; in addition, publication bias can affect these results. Further studies with larger sample sizes and a better distinction between virgin $v$. ordinary olive oil with respect to the risk of $\mathrm{CHD}$ are needed.

\section{Supplementary material}

To view supplementary material for this article, please visit http://dx.doi.org/10.1017/S0007114514000713

\section{Acknowledgements}

The present meta-analysis was financially supported by the official funding agency for Biomedical Research of the Spanish Government, Instituto de Salud Carlos III, through grants provided to research networks (RTIC G03/140 and RTIC RD 06/0045), and specific grants from Fondo de Investigación Sanitaria-Fondo Europeo de Desarrollo Regional (PI07/0240 and PI1002658), the Spanish Ministry of Health, Plan Nacional sobre Drogas, Programa 231-A Grant 2010/087 and CIBER Fisiopatologia de la Obesidad y Nutricion (CIBERobn) CB12/03/30017. The funding sources had no role in the design and conduct of the study; collection, management, analysis and interpretation of the data; and preparation, review or approval of the manuscript.

The authors' contributions were as follows: M. A. M.-G. and L. J. D. were responsible for the initial plan, study design, conducting the study, data interpretation and manuscript drafting; M. A. M.-G. and M. D.-R. were responsible for the statistical analysis; M. A. M.-G. and L. J. D. were responsible for the data collection, data extraction and data interpretation; M. A. M.-G., L. J. D. and M. D.-R. were responsible for the data interpretation, manuscript drafting, critical revision of intellectual content and approval of the final version of the manuscript.

There are no conflicts of interests.

\section{References}

1. Keys A (1987) Olive oil and coronary heart disease. Lancet 8539, 983-984.
2. Willett WC, Sacks F \& Trichopoulou A (1995) Mediterranean diet pyramid: a cultural model for healthy eating. Am J Clin Nutr 61, Suppl. 6, 1402S-1406S.

3. Tracy SW (2013) Something new under the sun? The Mediterranean diet and cardiovascular health. $N$ Engl $J$ Med 368, 1274-1276.

4. Keys A, Menotti A, Karvonen MJ, et al. (1986) The diet and 15-year death rate in the seven countries study. Am J Epidemiol 124, 903-915.

5. Gjonça A \& Bobak M (1997) Albanian paradox, another example of protective effect of Mediterranean lifestyle? Lancet 350, 1815-1817.

6. Lopez-Miranda J, Perez-Jimenez F, Ros E, et al. (2010) Olive oil and health: summary of the II international conference on olive oil and health consensus report, Jaen and Cordoba (Spain) 2008. Nutr Metab Cardiovasc Dis 20, 284-294.

7. Huang CL \& Sumpio BE (2008) Olive oil, the Mediterranean diet, and cardiovascular health. J Am Coll Surg 207, $407-416$.

8. Covas MI, Konstantinidou V \& Fito M (2009) Olive oil and cardiovascular health. J Cardiovasc Pharmacol 54, 477-482.

9. Ruiz-Canela M \& Martinez-Gonzalez MA (2011) Olive oil in the primary prevention of cardiovascular disease. Maturitas 68, 245-250.

10. Jakobsen MU, O'Reilly EJ, Heitmann BL, et al. (2009) Major types of dietary fat and risk of coronary heart disease: a pooled analysis of 11 cohort studies. Am J Clin Nutr 89, 1425-1432.

11. Bertuzzi M, Tavani A, Negri E, et al. (2002) Olive oil consumption and risk of non-fatal myocardial infarction in Italy. Int $J$ Epidemiol 31, 1274-1277, author reply $1276-1277$.

12. Ferro-Luzzi A, James WP \& Kafatos A (2002) The high-fat Greek diet: a recipe for all? Eur J Clin Nutr 56, 796-809.

13. Alemany M (2011) The Mediterranean diet: a group of healthy foods, a type of diet, or an advertising panacea? (Spanish). Med Clin (Barc) 136, 594-599.

14. Estruch R, Ros E, Salas-Salvado J, et al. for the PREDIMED investigators (2013) Primary prevention of cardiovascular disease with a Mediterranean diet. $N$ Engl J Med 368, 1279-1290.

15. Moher D, Liberati A, Tetzlaff J, et al. (2009) Preferred reporting items for systematic reviews and meta-analyses: the PRISMA statement. BMJ 339, b2535.

16. Samieri C, Feart C, Proust-Lima C, et al. (2011) Olive oil consumption, plasma oleic acid, and stroke incidence: the Three-City Study. Neurology 77, 418-425.

17. Wells GA, Shea B \& O'Connell D, et al. (2013) The Newcastle-Ottawa Scale (NOS) for assessing the quality of nonrandomized studies in meta-analyses. http://www.ohri.ca/ programs/clinical_epidemiology/oxford.asp (accessed 23 December 2013).

18. Greenland S \& O'Rourke K (2008) Meta-analysis. In Modern Epidemiology, 3rd ed., pp. 658-659 [KJ Rothman, S Greenland and TL Lash, editors]. Philadelphia, PA: Lippincott Williams and Wilkins.

19. Higgins JPT \& Thompson SG (2002) Quantifying heterogeneity in a meta-analysis. Stat Med 21, 1539-1558.

20. Higgins JPT, Thompson SG, Deeks JJ, et al. (2003) Measuring inconsistency in meta-analyses. BMJ 327, 557-560.

21. Higgins JP (2008) Commentary: heterogeneity in metaanalysis should be expected and appropriately quantified. Int J Epidemiol 37, 1158-1160.

22. Thorlund K, Imberger G, Johnston BC, et al. (2012) Evolution of heterogeneity $\left(I^{2}\right)$ estimates and their $95 \%$ 
confidence intervals in large meta-analyses. PLOS ONE 7, e39471.

23. Duval S \& Tweedie R (2000) A non-parametric "trim and fill" method of assessing publication bias in meta-analysis. $\mathrm{J} \mathrm{Am}$ Stat Ass 95, 89-98.

24. Sterne JAC (editor) (2009) Meta-Analysis in Stata: An Updated Collection from the Stata Journal. College Station, TX: Stata Press.

25. Dilis V, Katsoulis M, Lagiou P, et al. (2012) Mediterranean diet and CHD: the Greek European Prospective Investigation into Cancer and Nutrition cohort. Br J Nutr 108, 699-709.

26. Kontogianni MD, Panagiotakos DB, Chrysohoou C, et al. (2007) The impact of olive oil consumption pattern on the risk of acute coronary syndromes: the CARDIO2000 case-control study. Clin Cardiol 30, 125-129.

27. Misirli G, Benetou V, Lagiou P, et al. (2012) Relation of the traditional Mediterranean diet to cerebrovascular disease in a Mediterranean population. Am J Epidemiol 176, 1185-1192.

28. Bendinelli B, Masala G, Saieva C, et al. (2011) Fruit, vegetables, and olive oil and risk of coronary heart disease in Italian women: the EPICOR Study. Am JClin Nutr 93, 275-283.

29. Fernández-Jarne E, Martinez-Losa E, Prado-Santamaria M, et al. (2002) Risk of first non-fatal myocardial infarction negatively associated with olive oil consumption: a casecontrol study in Spain. Int J Epidemiol 31, 474-480.

30. Buckland G, Travier N, Barricarte A, et al. (2012) Olive oil intake and $\mathrm{CHD}$ in the European Prospective Investigation into Cancer and Nutrition Spanish cohort. Br J Nutr 108, 2075-2082
31. Martinez-Gonzalez MA \& Gea A (2012) Mediterranean diet: the whole is more than the sum of its parts. BrJ Nutr $\mathbf{1 0 8}$ $577-578$

32. Jacobs DR Jr, Gross MD \& Tapsell LC (2009) Food synergy: an operational concept for understanding nutrition. $\mathrm{Am} \mathrm{J}$ Clin Nutr 89, 1543S-1548S.

33. VanderWeele TJ (2009) On the relative nature of overadjustment and unnecessary adjustment. Epidemiology 20, 496-499.

34. Trichopoulou A, Costacou T, Bamia C, et al. (2003) Adherence to a Mediterranean diet and survival in a Greek population. $N$ Engl J Med 348, 2599-2608.

35. Esposito K, Marfella R, Ciotola M, et al. (2004) Effect of a Mediterranean-style diet on endothelial dysfunction and markers of vascular inflammation in the metabolic syndrome: a randomized trial. JAMA 292, 1440-1446.

36. Estruch R, Martinez-Gonzalez MA, Corella D, et al. (2006) Effects of a Mediterranean-style diet on cardiovascular risk factors: a randomized trial. Ann Intern Med 145, 1-11.

37. Covas MI, Nyyssonen K, Poulsen HE, et al. (2006) The effect of polyphenols in olive oil on heart disease risk factors: a randomized trial. Ann Intern Med 145, 333-341.

38. Visioli F \& Bernardini E (2011) Extra virgin olive oil's polyphenols: biological activities. Curr Pharm Des 17, 786-804.

39. Oh K, Hu FB, Cho E, et al. (2005) Carbohydrate intake, glycemic index, glycemic load, and dietary fiber in relation to risk of stroke in women. Am J Epidemiol 161, 161-169.

40. Thompson SG \& Higgins JPT (2002) How should metaregression analyses be undertaken and interpreted? Statist Med 21, 1559-1573. 